\title{
KEPLER OBSERVATIONS OF THREE PRE-LAUNCH EXOPLANET CANDIDATES: DISCOVERY OF TWO ECLIPSING BINARIES AND A NEW EXOPLANET
}

\author{
Steve B. Howell ${ }^{1,8}$, Jason F. Rowe ${ }^{2,9}$, William Sherry ${ }^{3}$, Kaspar von Braun $^{4}$, David R. Ciardi ${ }^{4}$, Stephen T. Bryson $^{2}$, \\ John J. Feldmeier ${ }^{5}$, Elliott Horch ${ }^{6}$, And Gerard T. VAN Belle ${ }^{7}$ \\ ${ }^{1}$ National Optical Astronomy Observatory, 950 North Cherry Avenue, Tucson, AZ 85719, USA \\ ${ }^{2}$ NASA Ames Research Center, Moffett Field, CA 94035, USA \\ ${ }^{3}$ National Solar Observatory, 950 North Cherry Avenue, Tucson, AZ 85719, USA \\ ${ }^{4}$ NASA Exoplanet Science Institute, California Institute of Technology, 770 South Wilson Avenue Pasadena, CA 91125, USA \\ ${ }^{5}$ Department of Physics and Astronomy, Youngstown State University, Youngstown, OH 44555, USA \\ ${ }^{6}$ Southern Connecticut State University, New Haven, CT 06515, USA \\ ${ }^{7}$ European Southern Observatory, Karl-Schwarzschild-Str 2., 85478 Garching, Germany \\ Received 2010 August 20; accepted 2010 October 19; published 2010 November 30
}

\begin{abstract}
Three transiting exoplanet candidate stars were discovered in a ground-based photometric survey prior to the launch of NASA's Kepler mission. Kepler observations of them were obtained during Quarter 1 of the Kepler mission. All three stars are faint by radial velocity follow-up standards, so we have examined these candidates with regard to eliminating false positives and providing high confidence exoplanet selection. We present a first attempt to exclude false positives for this set of faint stars without high-resolution radial velocity analysis. This method of exoplanet confirmation will form a large part of the Kepler mission follow-up for Jupiter-sized exoplanet candidates orbiting faint stars. Using the Kepler light curves and pixel data, as well as medium-resolution reconnaissance spectroscopy and speckle imaging, we find that two of our candidates are binary stars. One consists of a late-F star with an early M companion, while the other is a K0 star plus a late M-dwarf/brown dwarf in a 19 day elliptical orbit. The third candidate (BOKS-1) is an $r=15 \mathrm{G} 8 \mathrm{~V}$ star hosting a newly discovered exoplanet with a radius of $1.12 R_{\text {Jupiter }}$ in a 3.9 day orbit.
\end{abstract}

Key words: binaries: eclipsing - brown dwarfs - planetary systems

\section{INTRODUCTION}

Slightly more than two years before the launch of the NASA Kepler mission (Borucki et al. 2010a), a 40 night photometric variability study was undertaken in the Kepler field of view (Howell 2008). The Burrell Schmidt Optical Kepler Survey (BOKS) consisted of imaging a $1.25 \mathrm{deg}^{2}$ patch of sky centered on the open cluster NGC 6811 and was aimed at the detection of variable stars and exoplanet transits. Data were obtained using 3 minutes Sloan Digital Sky Survey (SDSS) $r$-band exposures with a few $V$-band exposures to provide color information and relate sources to other existing catalogs of stars in the field. The BOKS survey magnitude range is $r=14-20$ with over 35,000 point sources being measured and having light curves produced. Full details of the BOKS survey and our exoplanet transit candidate detection procedures are presented in Feldmeier et al. (2010).

This paper describes Kepler observations of three exoplanet candidate stars initially identified in the BOKS survey as having light curves suggestive of transit events. The three candidates had their discovery light curves and initial vetting described in Feldmeier et al. (2010). Since that time, ground-based reconnaissance spectra and high-resolution imaging have been obtained and the sources were observed by Kepler during Quarter 1 (Q1) operation. The transit events (initially discovered in the ground-based survey) were confirmed in the Kepler data. The near-continuous observations and high photometric precision of

\footnotetext{
8 Visiting Astronomer, Kitt Peak National Observatory, National Optical Astronomy Observatory, which is operated by the Association of Universities for Research in Astronomy (AURA) under cooperative agreement with the National Science Foundation.

9 NASA Postdoctoral Program Fellow.
}

Kepler have enabled us to quantify the observed events and to understand the candidate systems. While our analyses presented herein do not provide, by themselves, complete solutions for the non-exoplanet systems, they are nevertheless easily characterized as false positives. Additional ground-based spectroscopy, in the future, could be used to fully describe the non-exoplanet systems.

Our goal in this paper is to use the powerful constraints available from the analysis of the Kepler light curves and image data coupled with high-quality medium-resolution spectroscopy and high-resolution images to eliminate false positives and provide high confidence identification. This type of analysis and confirmation will be used in general for Kepler exoplanet candidates that are large (Jupiter-like planets of which $>100$ are currently known; Borucki et al. 2010b) and have SDSS $r$ magnitudes fainter than $\sim 13.5$. The large number of candidates and the relative faintness of their host stars essentially eliminate the ability to obtain enough large aperture high-resolution spectra to use for planet mass determination. Thus, the statistical value of Kepler results for large planets orbiting faint stars will rely on high levels of false positive elimination, and not on radial velocity measurements of the planet masses.

False positive elimination begins with a number of steps involving Kepler data itself. Using pipeline produced light curves (Jenkins et al. 2010a), and image data products (Bryson et al. 2010), candidates with at least three consistent events have transit models fit to their phase folded light curves. Additionally, detailed analysis of a number of aspects of the light curves, using difference imaging, and searching for centroid position shifts in the Kepler pixel data during transit, are also conducted. The details of the torturous false positive elimination path the Kepler data follow prior to ground-based observations is discussed in 

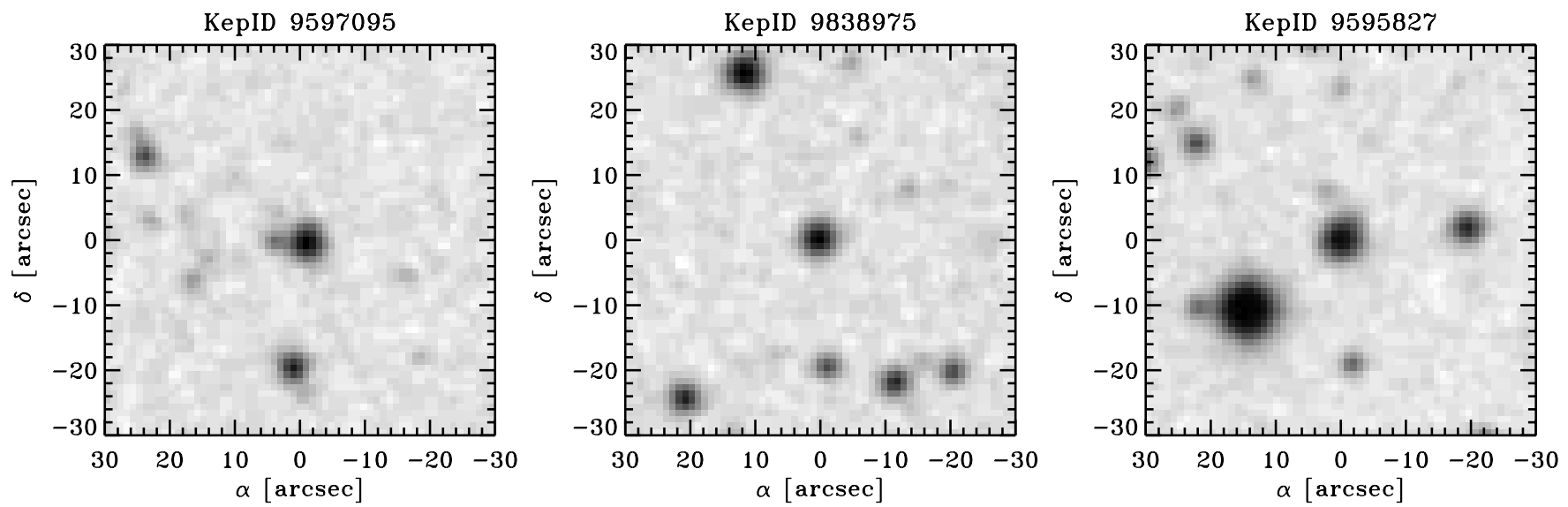

Figure 1. Finder charts for the three BOKS stars discussed in this paper. These charts show POSS2 Red images produced from the Digitized Sky Survey.

Table 1

KIC Photometry

\begin{tabular}{lccccccccc}
\hline \hline KepID & $\begin{array}{c}\text { R.A. } \\
\text { J2000 }\end{array}$ & $\begin{array}{c}\text { Decl. } \\
\text { J2000 }\end{array}$ & $\begin{array}{c}r \\
(\mathrm{mag})\end{array}$ & $\begin{array}{c}g \\
(\mathrm{mag})\end{array}$ & $\begin{array}{c}i \\
(\mathrm{mag})\end{array}$ & $\begin{array}{c}z \\
(\mathrm{mag})\end{array}$ & $\begin{array}{c}J \\
(\mathrm{mag})\end{array}$ & $\begin{array}{c}H \\
(\mathrm{mag})\end{array}$ & $\begin{array}{c}K s \\
(\mathrm{mag})\end{array}$ \\
\hline 9597095 & 194118.86 & +461606.2 & 15.90 & 16.53 & 15.69 & $\ldots$ & $14.91 \pm 0.04$ & $14.69 \pm 0.06$ & $14.69 \pm 0.11$ \\
9838975 & 194008.04 & +463600.5 & 16.10 & 16.83 & 15.86 & 15.69 & $14.65 \pm 0.03$ & $14.17 \pm 0.04$ & $13.96 \pm 0.05$ \\
9595827 & 193927.72 & +461709.1 & 15.06 & 15.69 & 14.89 & 14.80 & $13.93 \pm 0.03$ & $13.55 \pm 0.02$ & $13.47 \pm 0.04$ \\
\hline
\end{tabular}

Notes. Magnitude uncertainties are $\sim 0.02 \mathrm{mag}$ for $r, i, g$, and $\sim 0.03 \mathrm{mag}$ for $z$.

Batalha et al. (2010) and Borucki et al. (2010b). Only after these steps have been passed are ground-based observations brought into play, especially spectroscopy and high-resolution imaging.

The three stars are referred to herein by their Kepler identification number (KID) as given in the Kepler Input Catalog (KIC; Batalha et al. 2010). We present finding charts for each object in Figure 1 and coordinates and photometric information for them in Table 1. From our detailed analysis of the Kepler light curves and ancillary data and additional ground-based observations described below, we find that two of the original exoplanet candidates identified in the BOKS survey are eclipsing binaries while the third is found to be a true exoplanet system.

Sections 2-4 deal with our observations using Kepler, groundbased spectroscopy, and high-resolution speckle imaging. Section 5 presents conclusions for each candidate based on the observations and data analysis, while Section 6 concludes the paper.

\section{KEPLER PHOTOMETRIC OBSERVATIONS}

The Kepler mission and its photometric performance since launch are described in Borucki et al. (2010b), while the CCD imager on-board Kepler is described in Koch et al. (2010) and Van Cleve (2008). The observations used herein consist of data covering a time period of 32 days, obtained during Kepler's Q1 operation (2009 May-June corresponding to HJD 2454964 to 2454998). The Kepler photometry of our three stars are presented in Figures 2-4. The photometric observations were reduced using the Kepler mission data pipeline (Jenkins et al. 2010a) and then passed through various consistency checks and exoplanet transit detection software as described in Van Cleve (2009). Normalized and phase-folded light curves were then produced for each of the three BOKS exoplanet candidates. The transit events in the phased light curves were modeled in an effort to understand the transiting companion, both from the primary transit event and the secondary eclipse, if observed. Two of the candidates (KIC 9838975 and KIC 9597095) show clear evidence for a secondary eclipse and are consistent with being eclipsing binaries. The remaining candidate (KIC 9595827) is revealed to be a bona fide exoplanet.

\subsection{Transit Fits}

The Kepler photometric time series are initially fit to a transit (when the companion moves in front of the star) model to determine the scaled radius $\left(R_{p} / R_{\star}\right)$, scaled semimajor axis $\left(a / R_{\star}\right)$, impact parameter $(b)$, and the period $(P)$ and epoch $\left(T_{0}\right)$ of the companion. Our transit model uses the analytic formulae of Mandel \& Agol (2002) for a nonlinear limb-darkening law and best fits are found using a Levenberg-Marquardt algorithm (Press et al. 1992). Limb-darkening coefficients were adopted from fits to Atlas 9 spectra (Sbordone et al. 2004) convolved with the Kepler bandpass (A. Prsa 2010, private communication). We model the occultation (when the companion moves behind the star) by computing the fraction of the companion occulted by the star as a function of the star-planet projected distance and fitting for the brightness of the companion relative to the star. We assume the planet is a uniformly illuminated disc during occultation. In cases where we detect a significant occultation we allow for eccentric orbits and appropriately adjust the depth of the transit model to account for dilution. Uncertainties for the model parameters were derived from a Markov-Chain Monte Carlo (MCMC) analysis (Ford 2005). We refer the reader to Koch et al. (2010) for a full description of the transit modeling procedure.

We also compute an odd-even transit depth and occultation depth statistic that provide useful diagnostics in the identification of stellar binaries. The odd-even statistic determines the significance of a change of depth of odd and even-numbered 

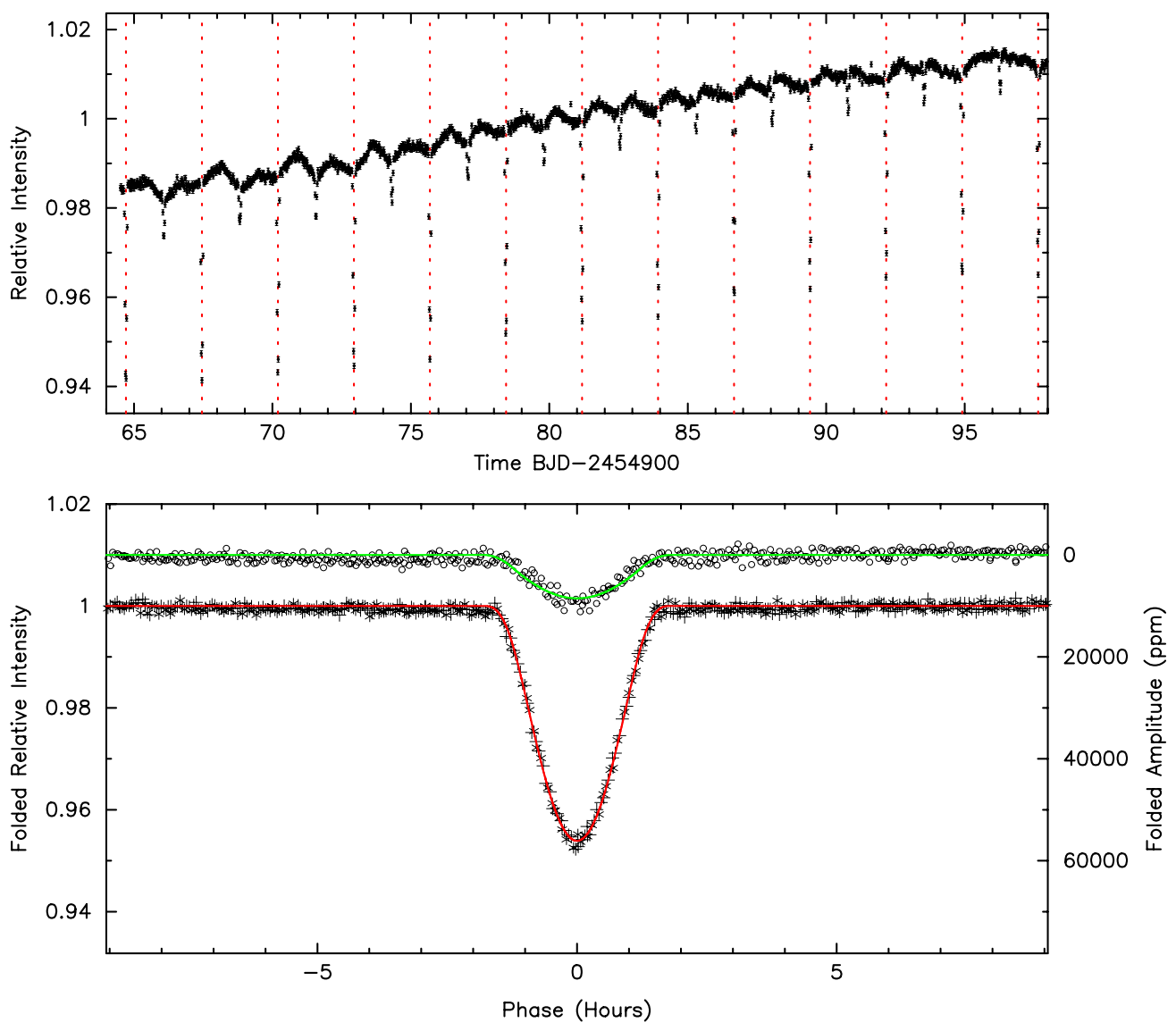

Figure 2. Kepler light curve for KID 9597095. The top panel shows the normalized Kepler light curve while the bottom panel shows this light curve phase folded near transit (phase 0.0 - crosses) and near phase 0.5 (circles). The plot has two vertical axes scales, the left one is for the transit depth (black crosses), while the right scale is for the secondary eclipse (gray circles). This object is an eclipsing binary.

transits. If the transit depths are found to be changing in such a fashion, then we have strong evidence that we are seeing a stellar binary where the two components have slightly different surface flux densities and the true orbital period is twice as long. The odd-even effects were measured at $0.7 \sigma, 1.6 \sigma$, and $0.5 \sigma$ confidence levels for KIC 9595827, KIC 9838975, and KIC 9597095, respectively. No significant odd-even effect was found for the three candidates described in this paper.

The occultation depth statistic attempts to measure the significance of an occultation of the companion. The search is done by first removing photometric measurements during transit and then phasing the remaining data at the orbital period and computing the maximum displacement of a segment of phased data with a length equal to the transit duration. No displacements are found for KIC 9595827 (the largest displacement had a $0.7 \sigma$ significance) level, while strong displacements were identified for KIC 9597095 and KIC 9838975.

The scaled semimajor axis is related to the mean density of the primary star and companion by

$$
\left(\frac{a}{R_{\star}}\right)^{3} \frac{\pi}{3 G P^{2}}=\frac{\left(M_{\star}+M_{p}\right)}{\frac{4 \pi}{3} R_{\star}^{3}} .
$$

If $M_{\star} \gg M_{p}$, then it is a simple matter to estimate the mean stellar density $\left(\bar{\rho}_{\star}\right)$ of the primary star and deduce its stellar parameters by matching to a set of theoretical stellar evolution models with an independent measurement of the stars' effective temperature (Sozzetti et al. 2007). For KIC 9838975 and KIC 9597095, the depth of the occultation is used to estimate the temperature of the companion by assuming the companion radiates as a blackbody and correcting for the Kepler bandpass. We estimate temperatures for the companion of $\sim 2700$ and $\sim 4000 \mathrm{~K}$, respectively. Such temperatures suggest companions with masses of $\sim 0.1$ and $\sim 0.5 M_{\odot}$. For a Jupitermass companion an error of $0.02 \%$ will be incurred on the measurement of $\bar{\rho}_{\star}$, a $0.1 M_{\odot}$ companion would skew our estimate of $\bar{\rho}_{\star}$ by $\sim 2 \%$, and a $0.5 M_{\odot}$ companion would induce a systematic error of $41 \%$ on $\bar{\rho}_{\star}$. It should also be noted that we have assumed a circular orbit for KIC 9595827. Should the companion exist in an eccentric orbit, our estimate of the scaled semimajor axis could exhibit large systematic errors (cf., Tingley et al. 2010).

We adopt estimates for the masses of the companions listed above and calculate $\bar{\rho}_{\star}$. For KIC 9597095, we also carry through an error of $10 \%$ to account for our uncertainty in the mass of the companion. We then proceed to determine the stellar parameters of the primary stars by matching $T_{\text {eff }}$ (see Table 2) with an adopted error of $150 \mathrm{~K}$ with the mean stellar density to the Yonsei-Yale stellar evolution tracks (Demarque et al. 2004) to calculate the range of stellar parameters consistent with the observation (e.g., Borucki et al. 2010b). This calculation produces a Markov Chain of 100,000 samples of $M_{\star}, R_{\star}$ pairs. These values are then used to determine new fits for $T_{0}, P$, $R_{p}$, orbital inclination $(i)$, and occultation depth as well as uncertainties in our model fits to light curves as listed in Table 3. 

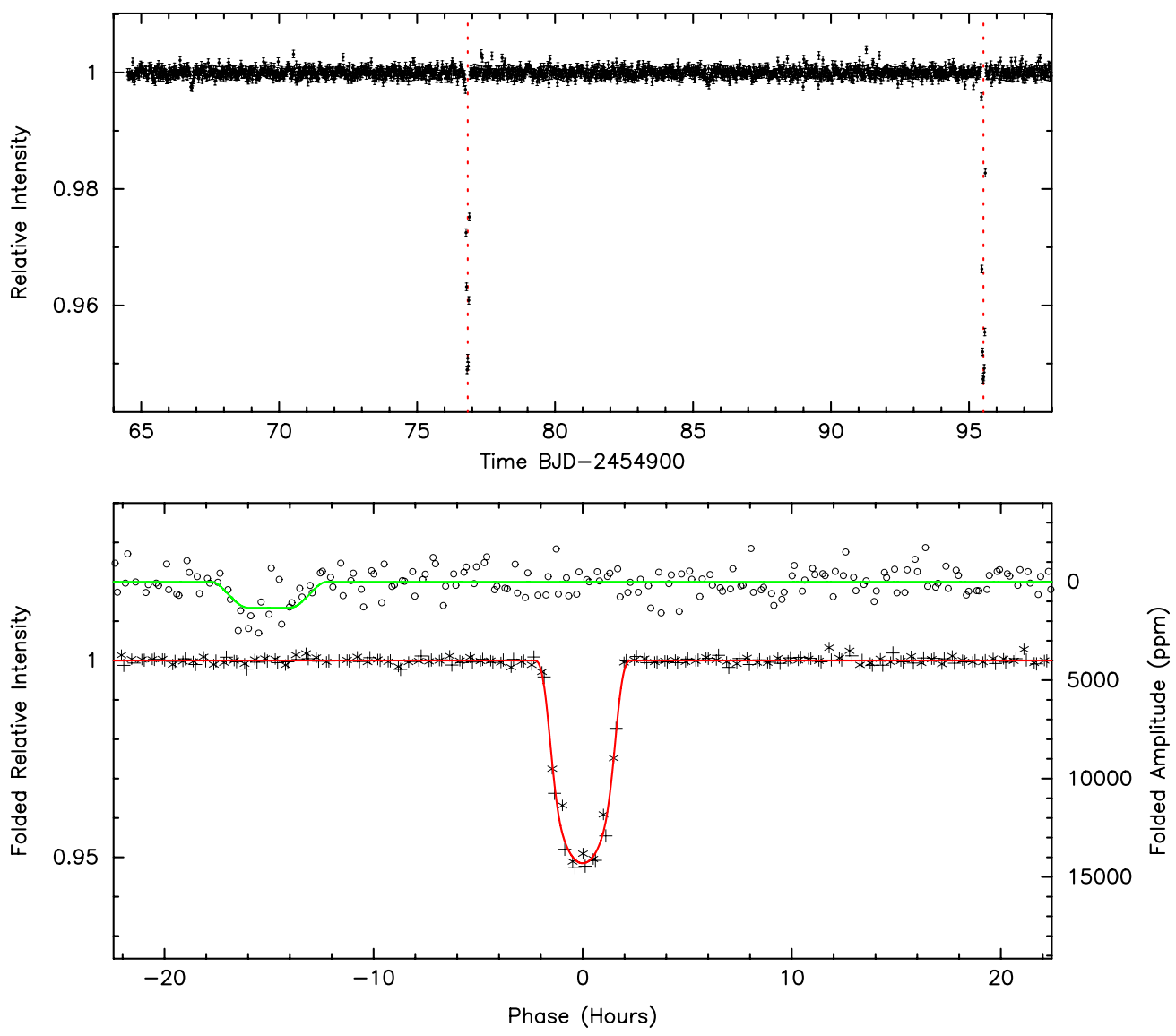

Figure 3. Kepler light curve for KID 9838975. See the caption of Figure 2. The scale for the occultation has been multiplied by a factor of 5 relative to the transit. The plot has two vertical axes scales: the left one is for the transit depth (black crosses), while the right scale is for the secondary eclipse (gray circles). This object is an eclipsing binary.

\subsection{Image Analysis}

Examination of the Kepler pixel images within the aperture of an exoplanet candidate star provides powerful extra constraints that can eliminate false positives. The stars KIC 9597095 and KIC 9838975 have already been eliminated as false positives for exoplanet candidates by virtue of their Kepler light curves alone. However, the exoplanet candidate KIC 9595827, not shown to be a false positive event based on the folded light curves and transit models discussed above, can be subjected to additional testing by making use of the downloaded image pixel data. The use of Kepler image pixel data for false positive elimination is described in Batalha et al. (2010). Details of initial image centroiding and difference image use are discussed by Jenkins et al. (2010b) and make for additional methods by which false positives can be identified. We have improved on those techniques as discussed below.

The pixel image aperture containing KIC 9595827 is shown in Figure 5 (top two panels) and is seen to contain 24 pixels in a $6 \times 4$ arrangement. Formation of these two images is done by first detrending and then folding the pixel time series and finally averaging over several in-transit ( 5 in this case) and out-of-transit (20) points. This improvement from the original analysis provides more robust images. The small change in flux of the target star between in and out of transit is too small to be noted here given the image stretch and the fact that a Kepler star is purposely slightly out of focus with an FWHM value near 4 arcsec (see Bryson et al. 2010). Since each pixel is 3.98 arcsec in size (Koch et al. 2010), the total aperture used here is 24 by 16 arcsec and is seen to contain our target star and a piece of a close companion. This brighter neighbor star can also be seen in Figure 1. The central source seen in Figure 5 is our target, KIC 9595827, while the other source, only partially in the aperture (at 1090, 552), is KIC 9595844 with $r \sim 13$. This star has its own Kepler light curve and shows no variation consistent with the transit observed in KIC 9595827. Its constant third light contribution to the total aperture flux is responsible for, and consistent with, the observed very small centroid shift seen in KIC 9595827 during transit $(-0.43 \pm 0.06,-0.99 \pm$ 0.09 millipixels).

Making use of Kepler pixel images for KIC 9595827 when in and out of transit, we can form a difference image and check to see if the target star is indeed the one changing in flux during the transit. We note in Figure 1 an additional close, faint companion to KIC 9595827 that will be well blended with the target star in the Kepler aperture. This star is 2.5-3 mag fainter than the primary object and would, if an eclipsing binary, have to have an eclipse depth of $14 \%$ in order to mimic the observed transit. This change in light would easily be observed in the difference image but is not seen. The bottom left panel of Figure 5 shows the difference image and reveals that indeed only KIC 9595827 varies during the transit.

A final and very informative use we can make from the pixel data is to produce individual pixel time series light curves. These are shown in Figure 6 in the same pixel arrangement as that of the Kepler pixel images in Figure 5. The transit event is clearly 

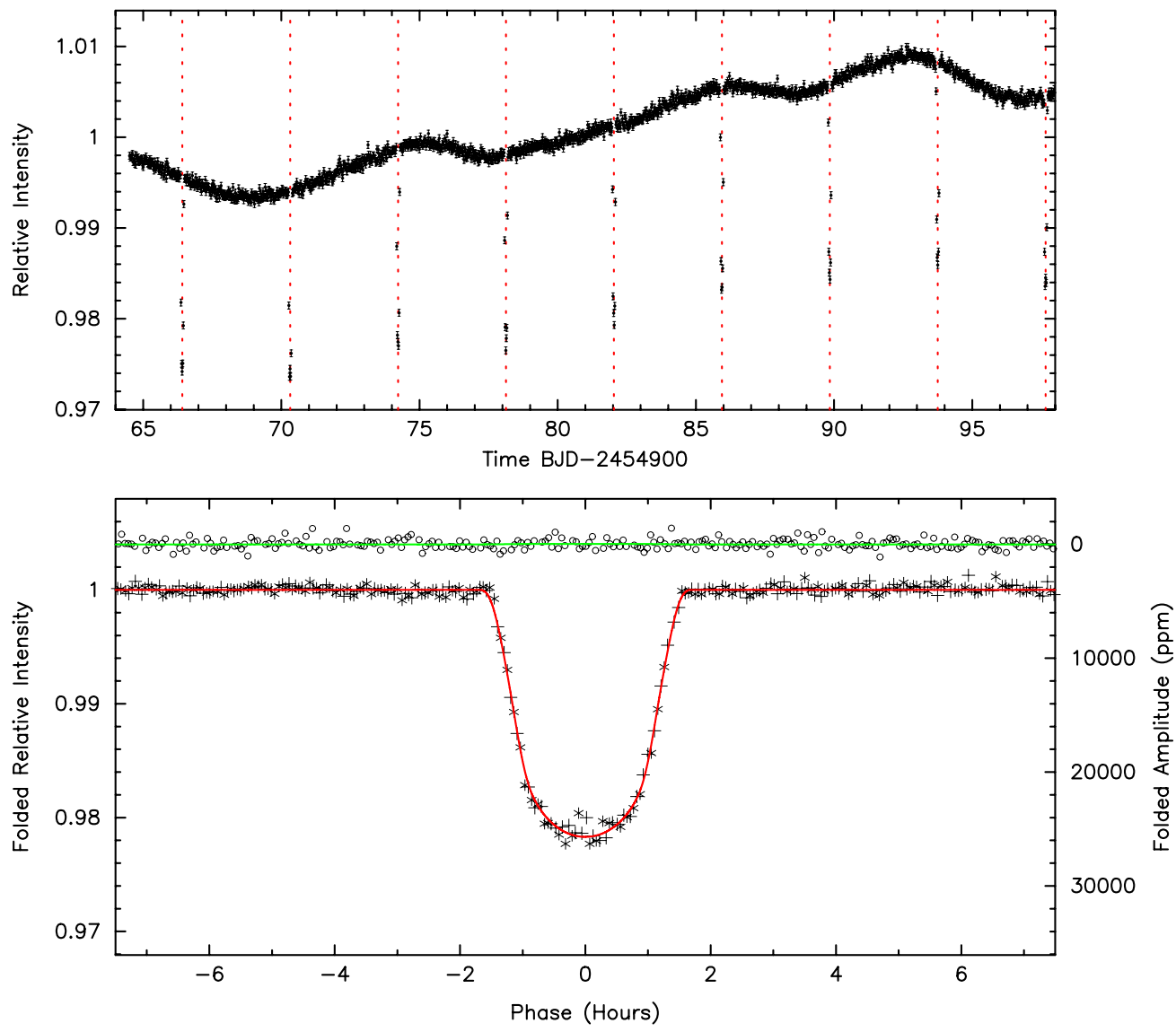

Figure 4. Kepler light curve for KID 9595827. See the caption of Figure 2. Note the wiggles in the light curve at the bottom of the transit indicative of starspots on the stellar surface. The plot has two vertical axes scales: the left one is for the transit depth (black crosses), while the right scale is for the secondary eclipse (gray circles). This object is a G8V star with a probable 1.12 Jupiter radius exoplanet in a 3.9 day orbit.

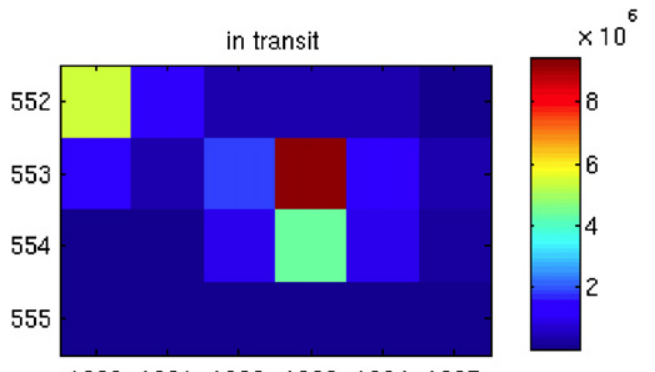

109010911092109310941095

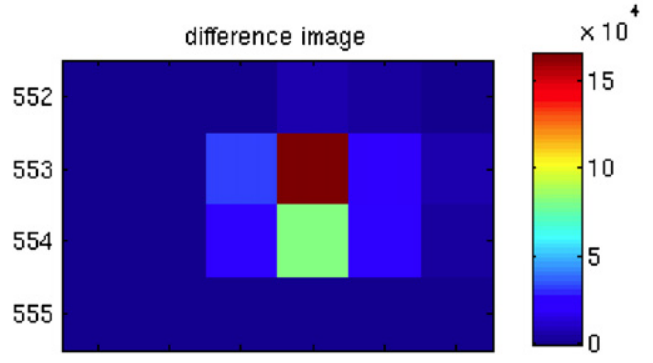

109010911092109310941095

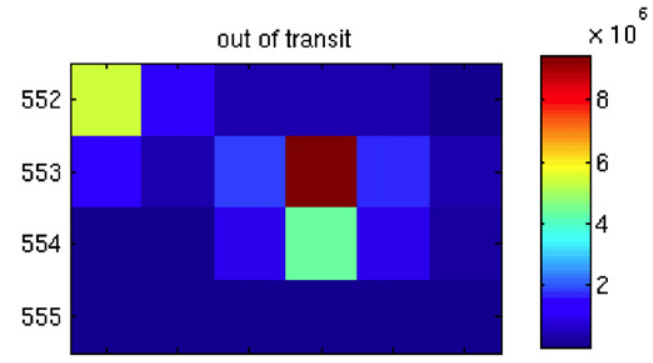

109010911092109310941095

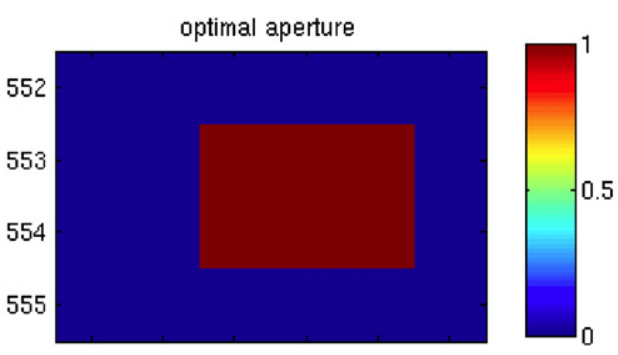

109010911092109310941095

Figure 5. Kepler pixel data images of KIC 9595827 (BOKS-1). The top panels show an in and out of transit image while the bottom left panel shows the difference image between the two. It is clear in the top two panels that a second star is (partially) present within the aperture for KIC 9595827 and its constant light accounts for the small but measurable centroid shift observed during transit. The difference image shows, however, that it is clearly only KIC 9595827 that varies during the exoplanet transit. 

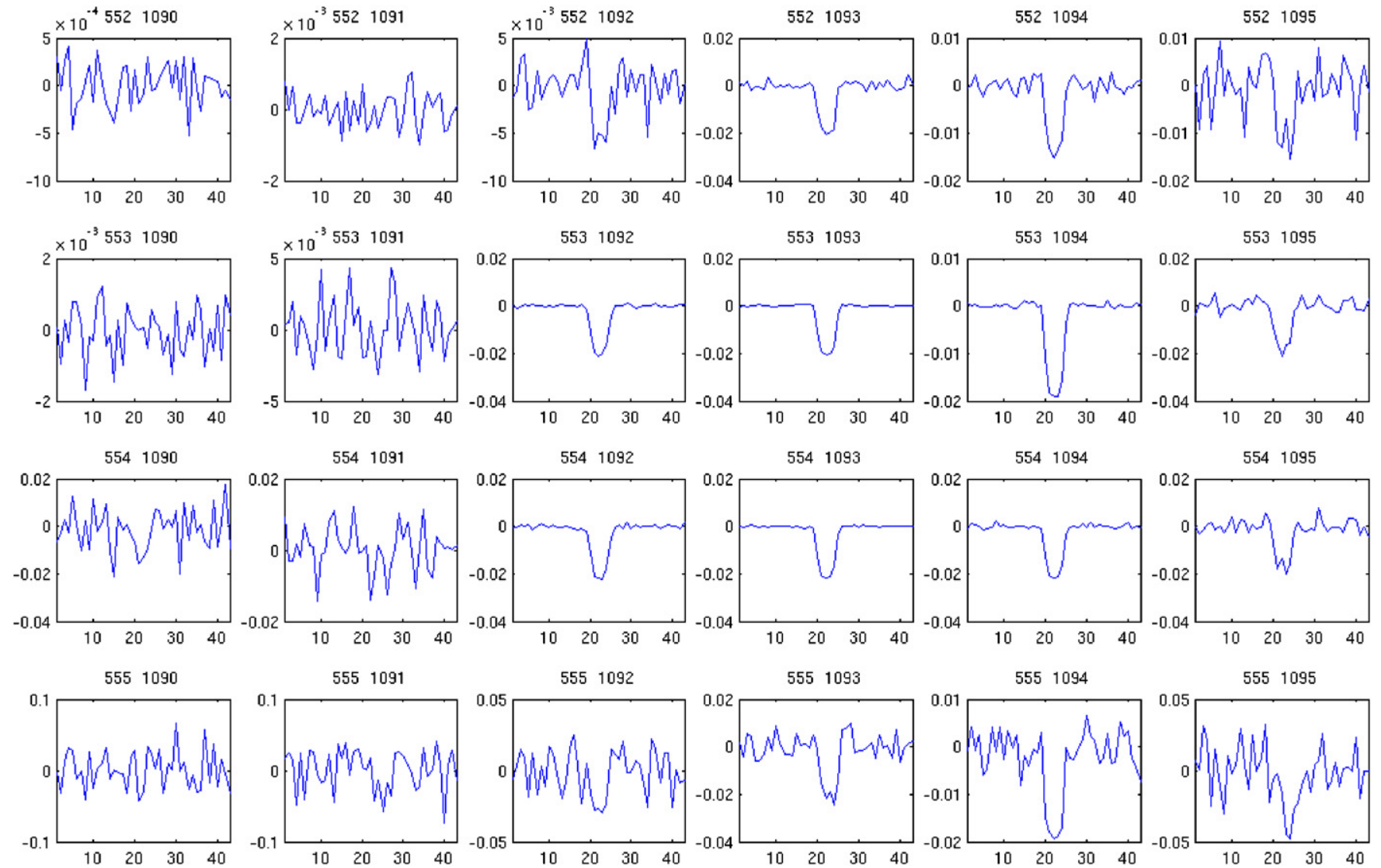

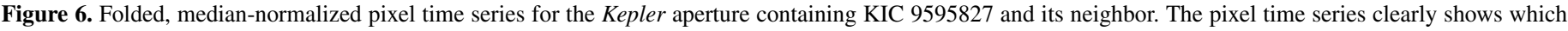
pixels contain the transit signal.

seen in the pixels containing KIC 9595827 but not observed in the brighter companion star partially in the aperture.

\section{SPECTROSCOPIC OBSERVATIONS}

Optical spectroscopy for our three candidate exoplanet stars was obtained using the Kitt Peak $2.1 \mathrm{~m}$ telescope and Gold Camera (GCAM) spectrograph and using the Kitt Peak $4 \mathrm{~m}$ telescope and RCSpec instrument. Spectra were obtained in 2008 June on both telescopes as well as in 2010 May on the $4 \mathrm{~m}$ to search for evidence of binarity and any spectral changes due to eclipsing binary motion, line shapes, and/or RV variations. The 2 m GCAM setup used a $3001 \mathrm{~mm}^{-1}$ grating (32) with a 1 arcsec slit to provide a mean spectral resolution of $2.4 \AA$ per resolution element across the full wavelength range. Both $4 \mathrm{~m} \mathrm{RCSpec}$ setups used a $6321 \mathrm{~mm}^{-1}$ grating (KPC-22b in second order) with a 1 arcsec slit to provide a mean spectral resolution of $1.6 \AA$ per resolution element across the full wavelength range. The spectra were reduced in the normal manner with observations of calibration lamps and spectrophotometric stars (obtained before and after each sequence) and bias and flat frames collected each afternoon.

The resulting spectra were compared with Morgan-Keenan (MK) standard stars digitally available in the "Jacoby Atlas" (Jacoby et al. 1984) and spectral types and luminosity classes were estimated by comparison to all stars in the atlas ( $\mathrm{O}$ to $\mathrm{M}$ and of luminosity classes I, III, IV, and V) via a standard $\chi^{2}$ fitting technique. Gaussian line fits to the strong spectral features were used to determine line centers, providing a velocity resolution of approximately one-tenth of a pixel across the full wavelength range in the $4 \mathrm{~m}$ spectra, or about $7 \mathrm{~km} \mathrm{~s}^{-1}$. Example spectra for
Table 2

Summary of Stellar Parameters

\begin{tabular}{ccccc}
\hline \hline KepID & $\begin{array}{c}\text { Temperature } \\
(\mathrm{K})\end{array}$ & $\begin{array}{c}\log (g) \\
\left(\mathrm{cm} \mathrm{s}^{-2}\right)\end{array}$ & $\begin{array}{c}\text { Metallicity } \\
(\text { solar }=0)\end{array}$ & $\begin{array}{c}\text { Spectral } \\
\text { Type }\end{array}$ \\
\hline 9597095 & 6400 & $\ldots$ & $\ldots$ & F8V-F9V \\
9838975 & 4967 & 4.802 & -0.511 & K0V \\
9595827 & 5545 & 4.724 & 0.22 & G8V \\
\hline
\end{tabular}

Notes. Temperature, $\log (g)$, and metallicity-derived photometrically from KIC data. Spectral type (and temperature for 9597095) derived from Kitt Peak spectra.

each object are shown in Figure 7. Table 2 presents our spectral type and effective temperature estimates based on the Kitt Peak observations, as well as other KIC-derived intrinsic properties for the candidate stars.

\section{SPECKLE OBSERVATIONS}

One of the most probable and devious false positive scenarios for photometric transit exoplanet surveys is the presence of a nearby companion star, either a real companion or a line-ofsight eclipsing binary. For a Jupiter depth transit event $(\sim 1 \%)$, a companion leading to a false positive must be within a few magnitudes of the target star as well as within about 1 arcsec. If a background star is fainter or further away, it cannot produce such a deep transit. The Kepler photometry alone eliminates much of the phase space here via centroiding measures of the pixel data in and out of the transit event. Typical direct imaging can identify companions as close as about $1-1.5$ arcsec and 

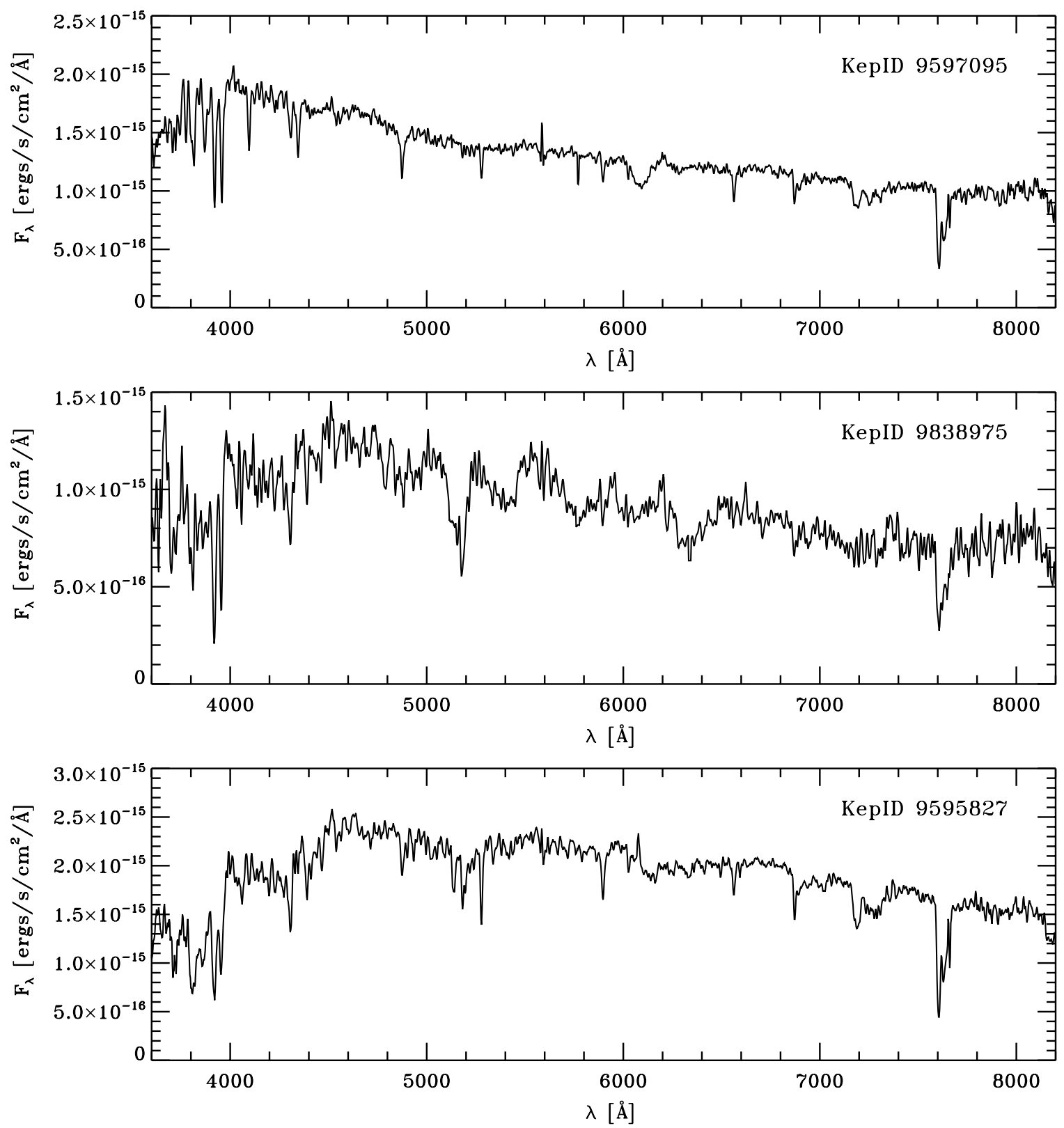

Figure 7. Example Optical Spectroscopy of the three exoplanet candidate stars obtained at the Kitt Peak $4 \mathrm{~m}$ telescope in 2008 June.

to perhaps 6-8 mag fainter. Medium-resolution spectroscopy can identify both orbital motion due to a stellar companion or direct evidence for two stars via a composite spectrum. However, for companions closer than 1 arcsec and to about 5-6 mag fainter, optical speckle observations are generally required to fully eliminate possible confounding sources. Techniques as deblending the images and fitting triple star scenarios are very powerful as well and have been successfully applied to exoplanet transit systems (DECPHOT, Weldrake et al. 2008; BLENDER, Torres et al. 2010).

Speckle observations form a major part of the Kepler mission false positive elimination strategy and are fully described in Horch et al. (2009) and Howell et al. (2010). We make use here of the Differential Speckle Survey Instrument, a recently upgraded speckle camera described in Horch et al. (2010) that provides simultaneous observations in two filters. The speckle instrument uses two identical EMCCDs and generally observes in " $V$ " and " $R$ " bandpasses, where " $V$ " has a central wavelength of $5620 \AA$ and an FWHM $=400 \AA$ and " $R$ " has a central wavelength of $6920 \AA$ and an FWHM $=400 \AA$. Our speckle observations of KID 9595827 reported here were obtained on 2010 June 21 and 22 UT with the WIYN 3.5 m telescope located on Kitt Peak. On both nights we obtained 8 sets of 1000 simultaneous " $V$ " and " $R$ " images each using 40 and $80 \mathrm{~ms}$ frame times, respectively. KID 9595827 , at Kepmag $=15.1$, is a fairly faint and challenging speckle target as well as being far too faint for high-resolution spectroscopic observations. The sets of speckle images were, as usual, co-added in Fourier space and re-projected into real space to produce the final reconstructed images (see Tokovinin et al. 2010).

Figure 8 shows the final reconstructed " $R$ " speckle image of KID 9595827 from 2010 June 22 UT. The two images at the bottom of the plot are identical but presented with different image stretch values for ease of use and visual identification of any possible companions. The "cross" pattern in each image is an artifact of the reconstruction process. 

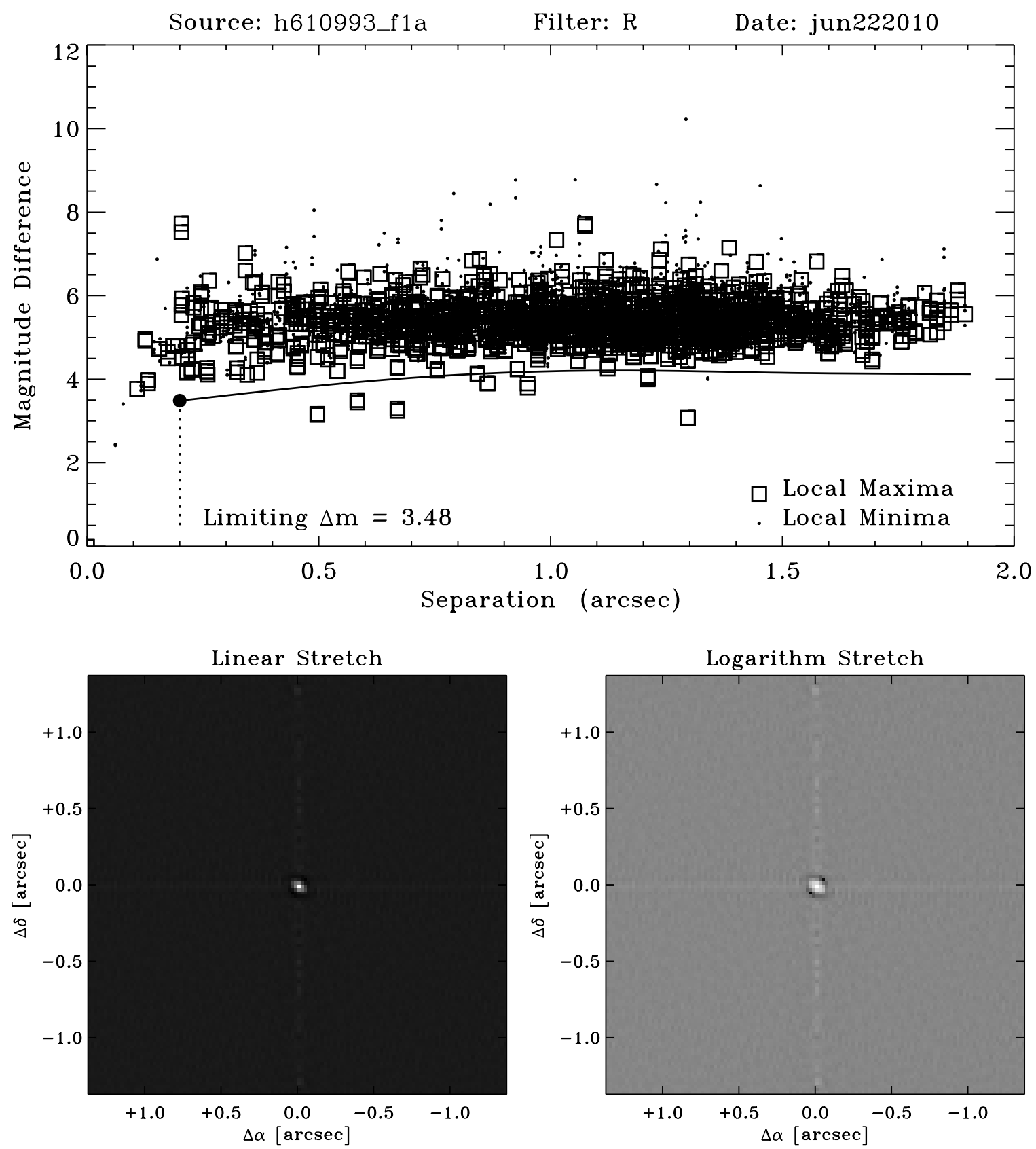

Figure 8. Speckle image of KID 9595827 showing that no line-of-sight or real companions exist from 0.05 to 1.8 arcsec of the star to a limit of 4.2 mag in $R$ and 5.0 mag in $V$ fainter than the star itself. The reconstructed images at the bottom of the plot have $\mathrm{N}$ up and $\mathrm{E}$ left. The horizontal line in the top plot shows the $5 \sigma$ detection limit for companions against the sky background (with three reconstruction artifact points lying below) and the vertical line at 0.2 arcsec is added to show the inner limit for conservative multi-fringe detections. See the text for details.

\section{DISCUSSION}

The three Kepler light curves presented in Figures 2-4 and their model fits tell us most of the story. The additional groundbased observations provide confirmation and some additional constraints for our three systems but the Kepler light curves alone get us about $90 \%$ of the way to a robust solution. The parameters from our model fits are listed in Table 3.

Table 1 provides KIC catalog information for the three stars. ${ }^{10}$ Table 2 has the derived stellar parameters for the three stars based on the catalog KIC colors and model fitting with the spectral type being determined from our spectra. The observed spectral type

\footnotetext{
${ }^{10}$ Note that the KIC is available at the MAST archives (http://archive.stsci.edu/).
}

is in very good agreement with the temperatures determined in the KIC. Table 3 summarizes the orbital elements for the companions. Since our ground-based survey field was centered near the open cluster NGC 6811, we were interested to see if any of the stars under study here were likely cluster members. None of the stars are at the cluster distance $(\sim 1200 \mathrm{pc})$ and, in addition, they all lie well outside the cluster core radius. Thus, we do not believe that these three stars are cluster members.

\subsection{KID 9597095}

The BOKS survey discovered this source as an $r=16$ variable showing a few percent exoplanet-like transit with a period near 2.7 days. The star, designated BOKS-r.45069, is revealed through optical spectroscopy (Figure 7) to be an F8-F9 main- 
Table 3

Transit Model Derived Parameters

\begin{tabular}{lccc}
\hline \hline \multicolumn{1}{c}{ Parameter } & 9597095 & 9838975 & 9595827 \\
\hline Period (days) & $2.74560 \pm 0.00003$ & $18.6929 \pm 0.00005$ & $3.90512 \pm 0.00005$ \\
$T_{0}(\mathrm{HJD} 2454900)$ & $64.7080 \pm 0.0002$ & $76.8324 \pm 0.0004$ & $66.4140 \pm 0.0002$ \\
$M_{\star}\left(M_{\odot}\right)$ & $1.21 \pm 0.03$ & $0.79_{-0.05}^{+0.03}$ & $0.95_{-0.05}^{+0.04}$ \\
$R_{\star}\left(R_{\odot}\right)$ & $1.43 \pm 0.01$ & $0.72 \pm 0.01$ & $0.86 \pm 0.02$ \\
$R_{p}\left(R_{\mathrm{J}}\right)$ & $5.9 \pm 0.5$ & $1.43_{-0.04}^{+0.02}$ & $1.11 \pm 0.02$ \\
$i(\mathrm{deg})$ & $79.4 \pm 0.5$ & $89.7_{-0.3}^{+0.2}$ & $89.8_{-0.4}^{+0.2}$ \\
$e$ & $0($ fixed $)$ & $0.15 \pm 0.02$ & $0(\mathrm{fixed})$ \\
$a(\mathrm{AU})$ & $0.0411 \pm 0.0003$ & $0.128 \pm 0.002$ & $0.0477 \pm 0.0007$ \\
$R_{p} / R_{\star}$ & $0.41 \pm 0.04$ & $0.2026_{-0.0011}^{+0.0006}$ & $0.1325_{-0.0003}^{+0.0005}$ \\
$\log g_{\star}$ & $4.21 \pm 0.01$ & $4.61 \pm 0.01$ & $4.54 \pm 0.01$ \\
Secondary depth (ppm) & $21033 \pm 3084^{\mathrm{a}}$ & $1173 \pm 228$ & $-33 \pm 65$ \\
$T_{\text {eff } p}(\mathrm{~K})$ & $4050 \pm 355$ & $2760 \pm 186$ & $<2200$ \\
\hline
\end{tabular}

Notes. ${ }^{\text {a }}$ Estimated depth if companion is fully occulted.

sequence star with an effective temperature near $6400 \mathrm{~K}$ and a distance estimate of $2500 \mathrm{pc}$. There is no evidence in our spectrum of a secondary star suggesting a large luminosity ratio. The Kepler light curve (Figure 2) shows an easily noted V-shaped primary eclipse and a clear secondary eclipse providing a deterministic diagnostic that this star is a grazing eclipsing binary. Knowing the primary star parameters, the eclipses allow the companion star and its orbit to be modeled. The secondary star has a radius of $5.9 R_{\text {Jupiter }}$ with an effective temperature near $4100 \mathrm{~K}$, consistent with a late $\mathrm{K}$ or early M-dwarf, having $0.83 \%$ of the luminosity of the F star primary. The orbital inclination is $79^{\circ}$ and the companion is likely heated by its close proximity to the $\mathrm{F}$ star. We note that the secondary eclipse depth in this system is $\sim 1 \%$, a level which is challenging but doable from the ground (cf., Weldrake et al. 2008). The lowmass companion star will be greatly out-shined by the F star in the optical and near-IR and a radial velocity solution for the binary will be required to fully determine the secondary star mass.

There is variability related to the orbital period of the companion that has a peak-to-peak amplitude of $\sim 1.8 \mathrm{mmag}$. Measuring the first two harmonics at 2.75 and 1.37 days gives amplitudes of 0.7 and $1.8 \mathrm{mmag}$, respectively. The second harmonic shows amplitude modulation over the timescale of the Kepler Q1 observations. If we interpret the cause of these interactions as ellipsoid distortions and Doppler boosting (Rowe et al. 2010; van Kerkwijk et al. 2010) then we can estimate a mass of approximately $0.4 M_{\odot}$.

\subsection{KID 9838975}

Discovered in the BOKS survey, this $r=16$ star showed an exoplanet transit-like event in its light curve but with only one transit observed, and its orbital period was unknown. Spectroscopy (Figure 7) determines the star, designated as BOKS-r.45069, to be a K0V ( \pm one subclass in spectral type) with an effective temperature near $5000 \mathrm{~K}$ and a distance of $\sim 1 \mathrm{kpc}$. The Kepler light curve (Figure 3 ) shows a U-shaped transit event as well as a clearly off-centered secondary eclipse suggestive of an elliptical orbit. The secondary eclipse in this system would be extremely difficult to observe from the ground as it has a depth near one-half of $1 \%$. Thus, this object would be extremely difficult to eliminate as an exoplanet using ground-based photometry alone. The companion causing the transit event has a radius of $1.4 R_{\text {Jupiter }}$ and a modeled effective temperature of $2760 \mathrm{~K}$. The orbital eccentricity is $\sim 0.1$, making the difference between aphelion and perihelion change by approximately $20 \%$. Thus, at an average distance from the $\mathrm{K} 0 \mathrm{~V}$ star of $\sim 0.13 \mathrm{AU}$, the companion is not close enough for irradiation to account for the observed temperature. At this low effective temperature, a low-mass red or brown dwarf is the most likely companion star in a near $90^{\circ}$ elliptical orbit with a period of 18.7 days. Additional Kepler observations and a radial velocity study should be able to easily confirm the elliptical nature of the orbit as well as provide a good mass estimate for the companion star.

\subsection{KID $9595827($ BOKS-1)}

The best exoplanet candidate from the BOKS survey, BOKS1 , is an $r=15$ variable showing an exoplanet transit-like event with a period near 4 days. Ground-based spectroscopy (Figure 7) shows the host star to be a G8V( \pm one subclass) with an effective temperature of $5500 \mathrm{~K}$ at a distance of $\sim 800 \mathrm{pc}$. Spectroscopic observations of this target obtained on two consecutive nights in 2008 June (roughly 0.5 apart in phase) and three nights in 2010 May reveal no statistically significant radial velocity trends at the $\sim 7 \mathrm{~km} \mathrm{~s}^{-1}$ level or larger. This limit rules out a companion star with a mass greater than $0.1 M_{\odot}$, i.e., virtually all dwarf stars.

Our speckle imaging was aimed at a search for background (or real) companions which may be the cause of the transit signal. To robustly estimate the background limit we reach in each reconstructed speckle image, we use the metrics derived from the top plot in Figure 8. If a companion is present, it will appear in the reconstructed image as a "point" like source, similar to the central source. As in a normal image, the value of pixels in the target and any companion star images will lie above the local "sky" background. If a nearby source appears and is approximately $3-5 \sigma$ above the background (the line in the top plot is a $5 \sigma$ limit from the mean of the distribution of background points) we will easily see it and can robustly measure its properties. If, however, a source is barely detected (say at $1.5 \sigma$ ), then we will just be able to note its presence, but would derive only approximate information for it. Local minima are also plotted mainly as a check to see if the minimum and maximum points are normally distributed (as would be the case for a well-produced reconstructed "sky").

Using our simultaneous two-color speckle camera we can detect companions, for bright targets, below the diffraction limit $(0.035 \operatorname{arcsec}$ at $5000 \AA)$ by detection of a single fringe in both cameras that provides the same solution. For fainter 
targets $(R \gtrsim 13.5-14.5)$ and good observing conditions, our inner detection limit ranges from 0 .'05 to 0 .' 15 depending on the magnitude difference of the companion. Thus, for multi-fringe images, a very conservative inner limit for robust detections is near 0.2 arcsec. Our high-resolution ground-based speckle observations allow us to eliminate any possible companion stars to $4.2 \mathrm{mag}$ in $R$ and $5.0 \mathrm{mag}$ in $V$ fainter and from $i$ $\sim 0.05$ to 1.8 arcsec of KID 9595827. Thus, the observed transit depth of $1.2 \%$ cannot be mimicked by a variable or eclipsing binary background star even if it showed a 50\% dimming. Taken together with the Kepler light curve model results, no companion star is present that can be the cause of the observed exoplanet transit event.

Image analysis of the Kepler pixel data for KIC 9595827 reveals that it is the source of variation within its aperture during the transit event and that its small centroid motion is consistent with the star itself dimming in the presence of a nearby constant light neighbor. Additionally, pixel time series light curves clearly reveal that the transit event is centered on our target star. No evidence from the image data suggests any explanation for the observed variation except as that of an exoplanet transit.

The Kepler light curve observations (Figure 4) show the typical U-shaped exoplanet transit and reveal no evidence for a secondary eclipse. The transiting body has a radius of 1.12 $R_{\text {Jupiter }}$ orbiting every 3.9 days at nearly $90^{\circ}$ to the plane of the sky. If this exoplanet is similar to other "hot Jupiters," it is likely to have a mass near one to a few Jupiter masses. The raw Kepler light curve shows evidence for a rotational modulation of the host star with a period near 17-18 days, and we note possible evidence of starspots seen as slight wiggles at the bottom of the exoplanet transit.

We tested the possibility of hierarchical triples, by adding a dilution factor to our models. The dilution factor accounts for inclusion of third light from an unseen stellar companion in the system. The effect is that the observed transit can be much shallower than reality. For example, if two stars of equal brightness are found within the Kepler aperture, the measured transit would need to be made twice as deep. We modeled the transit of KID 9595827 with dilution factors ranging from 0 to 0.7 , in increments of 0.05 , where 0.7 means $70 \%$ of the observed flux comes from an additional star within the photometric aperture. Comparing chi-squared values we find models with dilution factors greater than 0.1 are ruled out at the $99.97 \%$ confidence level. In particular, the egress and ingress of the models are incompatible with the size of the companion inferred from the depth of the transit. While there may be third light contamination due to a hierarchical triple scenario, the unseen companion must contribute less than $10 \%$ of the light in the photometric aperture. At such dilution factors, the planet radius would increase by $5 \%$ but still be compatible with being a bona fide planet.

In addition, the Kepler pixel data are not consistent with a nearly perfectly aligned (within $\sim 0.05$ arcsec) background eclipsing binary (BGEB) being the cause of the transit. To estimate the possibility of a chance alignment, we can take the mean local star density observed near KIC 9595827 (0.0122 stars $\operatorname{arcsec}^{-2}$ to $\left.r \sim 20\right)$ times the likelihood that a background star would be an eclipsing binary (1.2\% of stars are eclipsing binaries; Torres et al. 2010). The product of these values yields a probability of only $0.015 \%$ that a BGEB could be the cause of the transit event. If we assume a near circular orbit for the stars in any BGEB, we can estimate this probability in another way.
The observed transit time restricts the background population of confounding EBs to only G8 stars or earlier. Using the Besancon Galactic model, we calculate a stellar density of possible (G8 and earlier) pollutants to be $2 \mathrm{e}-4 \mathrm{stars} \operatorname{arcsec}^{-2}$. Taking the same likelihood as above that a background star could be an EB, this approach suggests only a $0.00024 \%$ chance that the transit could be caused by a BGEB. Given that these conservative estimates of a chance alignment of a BGEB or a hierarchical triple can be the cause of the observed transit event are essentially zero, we again conclude that KIC 9595827 (BOKS-1) does indeed harbor an exoplanet.

Kepler is still monitoring this source and revealing continuous transits leaving no doubt as to its periodic nature and constant transit shape and depth. Phase folding of all the data to date reveals no secondary eclipse eliminating essentially all possible binary star configurations. Future Kepler observations will hopefully detect the occultation allowing one to determine the companions orbit. At $r=15$, it is unlikely that high-precision radial velocities will soon be forthcoming for this system.

\section{CONCLUSIONS}

Kepler observations have provided unprecedented light curves for three pre-launch exoplanet candidate stars. Two of them, KID 9838975 and KID 9597095, are shown to be main sequence plus late-type dwarf/brown dwarf binaries, one harboring its companion in a 19 day elliptical orbit. We note that Kepler photometry was likely necessary in order to observe the secondary eclipse in KID 9838975 and may ultimately detect the occultation in KID 9595827 (BOKS-1). Such small dips would unlikely be distinguishable from the ground due to the necessary precision required over their duration and covering a number of occultations. This type of binary star light curve suggests a word of caution for ground-based exoplanet candidates, even those with well-determine light curves, as very small secondary eclipses or occultations would easily be missed. The two binary stars discovered here are ripe for radial velocity followup in order to determine a better mass and orbital characteristics for the low-mass companions.

We have presented a first attempt to fully utilize Kepler light curves and Kepler image analysis combined with ground-based follow-up observations to exclude false positive exoplanet candidates without high-resolution spectroscopic radial velocity measurements. The best pre-launch BOKS survey exoplanet transit candidate (BOKS-1) has been confirmed by this methodology to be a true exoplanet; a "hot Jupiter" having a radius of $1.12 R_{\text {Jupiter }}$ and an orbital period near 3.9 days. The Kepler light curves and pixel data analysis alone can provide a very robust method to eliminate false positives and confirm, at a high confidence level, Jupiter-size planets. However, ground-based follow-up observations provide additional information and confirmation of the Kepler results as well as placing hard constraints on the possible companions. This system at $r=15$ will likely need to await the next generation of larger telescope and/or a more sensitive radial velocity instrument before its exoplanet mass can be precisely determined.

We thank Brandon Tingley for his timely review and very helpful comments on our original manuscript. The authors thank the Kepler Science Office and the Science Operations Center personal, particularly Natalie Batalha, Jon Jenkins, and Tim Brown, for their dedicated effort to the mission and for providing us access to the science office data products. The ground-based observations reported herein were obtained at Kitt Peak National 
Observatory, National Optical Astronomy Observatory, which is operated by the Association of Universities for Research in Astronomy (AURA) under cooperative agreement with the National Science Foundation. The Kepler Science Team is thanked for their help and support of the mission and its scientific output. Kepler was selected as the 10th mission of the Discovery Program. Funding for this mission is provided by NASA.

\section{REFERENCES}

Batalha, N., et al. 2010, ApJ, 713, L103

Borucki, W., et al. 2010a, Science, 327, 977

Borucki, W., et al. 2010b, ApJ, 713, L126

Bryson, S. T., et al. 2010, ApJ, 713, L97

Demarque, P., Woo, J.-H., Kim, Y. -C., \& Yi, S. 2004, ApJS, 155, 667

Feldmeier, J., et al. 2010, AJ, in press

Ford, E. 2005, AJ, 129, 1706

Horch, E. P., Veillette, D. R., Baena Gallé, R., Shah, S. C., O’Rielly, G. V., \& van Altena, W. F. 2009, AJ, 137, 5057

Horch, E. P., Shamilia, C., Gomez, S. C., Sherry, W. H., Howell, S. B., Ciardi, D. R., Anderson, L. M., \& van Altena, W. F. 2010, AJ, in press

Howell, S. B. 2008, Astron. Nachr., 329, 259
Howell, S. B., Sherry, W. H., Horch, E. P., Ciardi, D. R., \& Everett, M. E. 2010, AJ, submitted

Jacoby, G. H., Hunter, D., \& Christian, C. 1984, ApJS, 56, 257

Jenkins, J. M., et al. 2010a, ApJ, 713, L87

Jenkins, J. M., et al. 2010b, arXiv:1001.0416

Koch, D., et al. 2010, ApJ, 713, L131

Mandel, K., \& Agol, E. 2002, ApJ, 580, 171

Press, W. H., Teukolsky, S. A., Vetterling, W. T., \& Flannery, B. P. 1992, Numerical Recipes in Fortran 77 (2nd ed.; Cambridge: Cambridge Univ. Press), 678

Rowe, J. F., et al. 2010, ApJ, 713, 150

Sbordone, L., Bonifacio, P., Castelli, F., \& Kurucz, R. L. 2004, MSAIS, 5, 93

Sozzetti, A., Torres, G., Charbonneau, D., Latham, D. W., Holman, M. J., Winn, J. N., Laird, J. B., \& O'Donovan, F. T. 2007, ApJ, 664, 1190

Tingley, B., Bonomo, A. S., \& Deeg, H. J. 2010, ApJ, submitted

Tokovinin, A., Mason, B. D., \& Hartkopf, W. I. 2010, AJ, 139, 743

Torres, G., et al. 2010, ApJ, in press

Van Cleve, J. 2008, Kepler Instrument Handbook (available at http://archive.stsci.edu/kepler/)

Van Cleve, J. (ed.) 2009, in Kepler Data Release Notes (available at http://archive.stsci.edu/kepler/)

van Kerkwijk, M. H., et al. 2010, ApJ, 715, 51

Weldrake, D. T. F., Bayliss, D. D. R., Sackett, P. D., Tingley, B. W., Gillon, M., \& Setiawan, J. 2008, ApJ, 675, L37 